ORIGINAL RESEARCH

N. Sato

M. Ota

A. Yagishita

Y. Miki

T. Takahashi

Y. Adachi

Y. Nakata

K. Sugai

M. Sasaki

\section{Aberrant Midsagittal Fiber Tracts in Patients with Hemimegalencephaly}

BACKGROUND AND PURPOSE: In hemimegalencephaly, MR imaging often reveals midsagittal bandlike structures between the 2 lateral ventricles. To determine whether these structures are aberrant midsagittal fibers, we retrospectively reviewed them on conventional MR imaging and prospectively examined them by diffusion tensor MR and fiber tract (FT) reconstruction imaging.

MATERIALS AND METHODS: We retrospectively reviewed conventional MR images of 26 consecutive patients with hemimegalencephaly by 2 neuroradiologists, focusing on abnormal midsagittal structures. The distance between the 2 anterior horns and widths of midsagittal bandlike structures were measured. Prospective analysis was performed in 7 consecutive patients with hemimegalencephaly examined for midsagittal aberrant fibers by diffusion tensor imaging, and cortical distribution areas of the fibers were observed.

RESULTS: The distance between the 2 anterior horns was wide $(>4 \mathrm{~mm})$ due to white matterintensity structures in 20 of 26 patients (76.9\%). Mid-sagittal bandlike structures were observed in 15 patients $(57.7 \%)$. Asymmetry of the fornices was detected in 7 patients $(26.9 \%)$, and both fornices were thickened in 7 (26.9\%) patients. On FT reconstruction, images showed that 4 of 7 patients with hemimegalencephaly had aberrant midsagittal fibers connecting frontal, occipital, or parietal lobes, bilaterally $(n=3)$ or ipsilaterally $(n=1)$. All 4 patients had increased width between the 2 anterior horns, and 3 of them exhibited midsagittal bandlike structures on conventional MR imaging. On the other hand, these MR imaging findings were not noted in 3 patients who did not have aberrant midsagittal fibers on diffusion tensor imaging.

CONCLUSIONS: Aberrant midsagittal FTs running intra- or interhemispherically do not infrequently exist in patients with hemimegalencephaly.

H emimegalencephaly is a rare but unique malformation characterized by enlargement of all or a part of a cerebral hemisphere. ${ }^{1-6}$ These morphologic, histologic, and MR imaging findings have previously been described. ${ }^{7-9}$ However, to our knowledge, no reports have focused on abnormal midsagittal structures. In most patients with hemimegalencephaly, the distance between the 2 anterior horns of the lateral ventricles is increased due to the presence of white matter-intensity regions, where a thin septum pellucidum is present in healthy subjects. In addition, the white matter-intensity region between the anterior horns continues posteriorly to form a bandlike structure between the 2 lateral ventricles and beneath the body of the corpus callosum. We speculated that the midsagittal bandlike structures may be aberrant white matter tracts, given their intensity and continuity.

Diffusion tensor imaging has opened up new possibilities of imaging of fiber tracts (FTs) in the brain by estimation of the whole diffusion tensor. Diffusion tensor imaging enables evaluation of the orientation of white matter fibers determined from the primary eigenvector of the diffusion tensor and can depict the 3D FT from directional information. ${ }^{10}$ Use

Received August 13, 2007; accepted after revision October 29.

From the Departments of Radiology (N.S., M.O., T.T., Y.A.) and Child Neurology (K.S., M.S.), Musashi Hospital, National Center of Neurology and Psychiatry, Kodaira, Tokyo, Japan; Department of Neuroradiology (A.Y.), Tokyo Metropolitan Neurological Hospital, Fucyu, Tokyo, Japan; Department of Diagnostic Imaging and Nuclear Medicine (Y.M.), Kyoto University School of Medicine, Kyoto, Japan; and Department of Radiology (Y.N.), Tokyo University School of Medicine, Bunkyo-ku, Tokyo, Japan.

Please address correspondence to Noriko Sato, MD, PhD, Department of Radiology, Musashi Hospital, National Center of Neurology and Psychiatry, 4-1-1 Ogawahigashi-cyo, Kodaira, Tokyo 287-8511, Japan; e-mail: snoriko@ncnp.go.jp

DOl 10.3174/ajnr.A0919 of the 3D tracking method has enabled successful identification of axonal projections. ${ }^{11}$

In this study, we retrospectively reviewed $M R$ imaging findings of patients with hemimegalencephaly, focusing on midsagittal structures, such as the distance between the anterior horns occupied by white matter-intensity regions; the existence of bandlike structures; and asymmetry or enlargement of the fornices. We also prospectively evaluated aberrant midsagittal fibers by diffusion tensor MR and FT reconstruction imaging and compared results with those of MR imaging.

\section{Materials and Methods}

\section{Patients and MR Imaging Examinations}

We first performed a retrospective MR imaging analysis. MR imaging findings were reviewed for 26 serial patients with hemimegalencephaly ( 19 males and 7 females; 11 weeks to 19 years of age; mean age, $1.6 \pm 4.6$ years). The patients were identified by medical records and had been hospitalized between January 1998 and December 2005 in 2 institutions. All patients had been hospitalized for intractable epilepsy, with detailed examinations performed to determine whether surgical treatment was indicated. The diagnosis was made on the basis of both clinical and imaging findings. The standard evaluation included detailed history and neurologic examinations, scalp electroencephalographic recording, technetium-Tc99m L-ethyl cysteinate dimmer single-photon emission CT, and MR imaging studies. We also prospectively performed MR imaging studies, including diffusion tensor sequences for 7 serial patients with hemimegalencephaly ( 6 males and 1 female; 2 months to 11 years of age; mean age, $4.3 \pm 4.4$ years) in our institution from March 2005 to November 2006. The standard evaluation for diagnosis was the same as that for the retrospective study. This study had appropriate ethics committee approval, and written informed consent was obtained from all patients. 

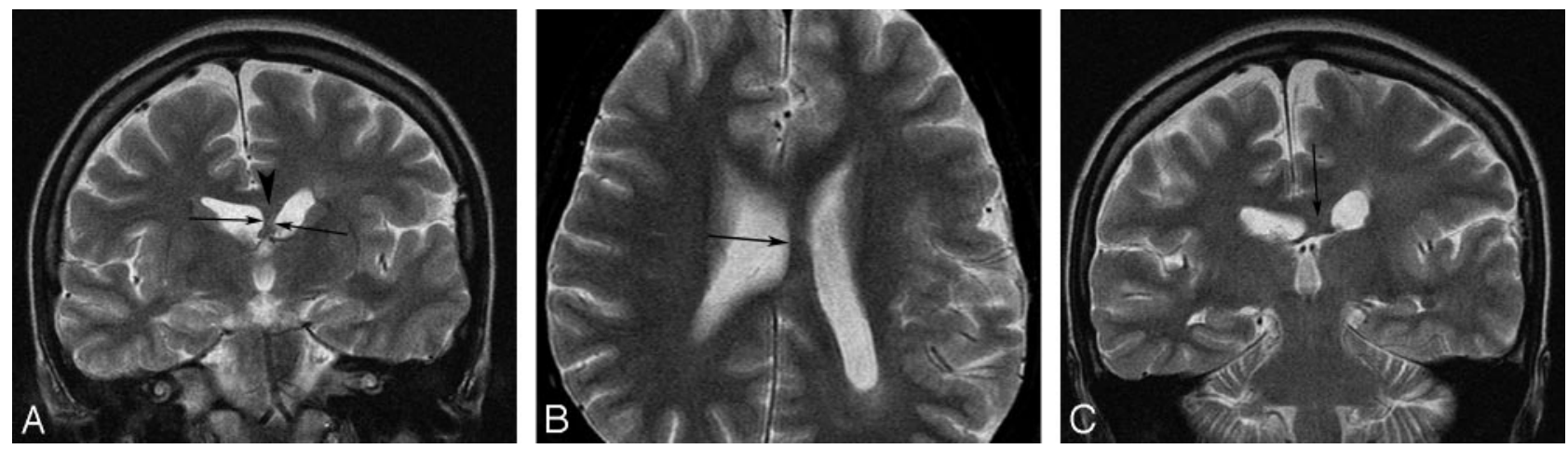

Fig 1. A 19-year-old female patient with left hemimegalencephaly evaluated in the retrospective analysis. A, T2-weighted image shows increased width (arrows) between the 2 anterior horns due to a white matter-intensity structure (arrowhead). $B$ and $C$, The white matter-intensity structure appears to extend posteriorly and to pass through the bodies of the lateral ventricles beneath the corpus callosum (arrow).

In a retrospective analysis, MR images were obtained on a $1 \mathrm{~T}$ (Magnetom Impact; Siemens, Erlangen, Germany) or 1.5T imager (Signa; GE Medical Systems, Milwaukee, Wis). Sequences included T1-weighted images (TR, 300-624 ms; TE, 9-15 ms; section thickness, 4-7 mm; intersection gap, 0.5-1.7 mm; matrix, $256 \times 224$ or $256 \times 256$; number of acquisitions, 2), T2-weighted images (TR, 4000-4200 ms; TE, 95-121 ms; section thickness, 3-6 mm; intersection gap, .05-1.7 mm; matrix, $256 \times 224$ or $256 \times 256$ or $512 \times 448$; number of acquisitions, 2 or 3 ), fluid-attenuation inversion recovery images (TR, 8000-10002 ms; TE, 2200-2500 ms; T1, 104-158 ms; section thickness, 5-6 mm; intersection gap, 1-2.5 mm; matrix, $256 \times 192$, or $256 \times 256$, or $512 \times 432$; number of acquisitions, 1 ), T2-weighted inversion recovery images (TR/TE/IR; TR, 4000-4210; TE, 120-150; TI, 32-85; section thickness, 3-4 mm; intersection gap, 0.3-2 mm; matrix, $256 \times 256$ or $512 \times 432$; number of acquisitions, 2 or 3 ), and 3D high-resolution sagittal T1-weighted fast low-angle images (TR, 1970 ms; TE, 3.9 ms; TI, 110 ms; sagittal thickness sections, $1.2 \mathrm{~mm}$; 144 sections per patient; matrix, $512 \times 228$; number of acquisitions, 1). Three different planes were obtained in all MR imaging examinations except 3 studies that included the axial and coronal planes.

In 7 patients with hemimegalencephaly, prospective MR imaging studies were performed on a $1 \mathrm{~T}$ imager. In addition to the conventional sequences of 3 different planes as described previously, diffusion tensor imaging was performed in the axial plane (TR, 10,100 ms; TE, $113 \mathrm{~ms}$; FOV, $230 \times 230 \mathrm{~mm}^{2}$; matrix, $128 \times 128$; 40 continuous transverse sections; section thickness, $3 \mathrm{~mm}$; no intersection gap). To enhance the signal intensity-to-noise ratio, the acquisition was repeated 5 times. Diffusion was measured along 12 noncollinear directions with the use of a diffusion-weighted $\mathrm{b}$ factor in each direction, with $700 \mathrm{~s} / \mathrm{mm}^{2}$; and 1 image was acquired without the use of a diffusion gradient.

\section{Interpretation of MR Imaging Findings}

All MR images were evaluated by 2 neuroradiologists (N.S. and A.Y., with 19 and 25 years of experience with MR imaging, respectively). Results were determined by consensus of the 2 readers. All serial images obtained for each patient were reviewed at the same time. We measured the distance between the inner walls of the anterior horns of the lateral ventricles at the narrowest point, which were opacified by white matter-intensity structures where the septum pellucidum was present in healthy subjects. When the cavum of the septum pellucidum was identified, the distance was measured at the solid portions excluding the cavities. The distance was considered wide when it was
$>4 \mathrm{~mm}$, due to the certainty and measurement errors. We also evaluated the presence or absence of midsagittal bandlike structures between the 2 lateral ventricles and beneath the body of the corpus callosum. When they were identified, we measured their thickness. We also evaluated the asymmetry and thickness of the fornices, which sometimes transited to the midsagittal bandlike structures.

\section{Diffusion Tensor Imaging Data Processing}

Raw diffusion tensor data were transferred to a workstation, diffusion tensor imaging datasets were analyzed by using DTIStudio software (Johns Hopkins University, Baltimore, Md; available at http:// cmrm.med.jhmi.edu), and 3D FTs were drawn on color maps. ${ }^{12}$ In axial, coronal, and sagittal sections, green was assigned to anteroposterior, red to left-right, and blue to craniocaudal directions. ${ }^{13,14}$ To demonstrate the aberrant midsagittal fibers passing anteroposteriorly through between the 2 anterior horns of the lateral ventricles (Fig. $1 A$ ), we used a region-of-interest approach. ${ }^{15}$ First, tracking was performed by using all pixels inside the brain, and results included in the manually defined regions of interest were assigned to the specific tracts associated with the regions of interest. ${ }^{16}$ We located the regions of interest in the green area between the anterior horns of the lateral ventricles on the coronal image, with attention paid not to include the red corpus callosum just above it. Fiber tractography was performed with the threshold value of fiber-tracking termination as FA $=0.2$ and a trajectory angle of $50^{\circ} .{ }^{17} \mathrm{We}$ observed the existence of midsagittal aberrant fibers. If an FT of a part of the fornix was drawn, it was not considered as aberrant fibers because the column of fornix is located inferior to the septum pellucidum. All fibers except for the fornix were admitted as aberrant fibers. When midsagittal aberrant fibers were detected, their cortical distribution areas and penetrating courses were observed.

\section{Results}

\section{Retrospective MR Imaging Findings for Mid-Sagittal Structures}

The distance between the 2 anterior horns was wide ( $>4 \mathrm{~mm}$ ) and occupied by white matter-intensity structures in 20 of the 26 patients $(76.9 \%)$ (Fig $1 A$ ). Thickness ranged from 2 to 18 mm (mean, $6.5 \pm 4.5 \mathrm{~mm}$ ). Mid-sagittal bandlike structures between the lateral ventricles and beneath the body of the corpus callosum were observed in 15 of the 20 patients (75\%) and in $57.7 \%$ of all patients (Fig $1 B,-C$ ). Their thickness ranged 

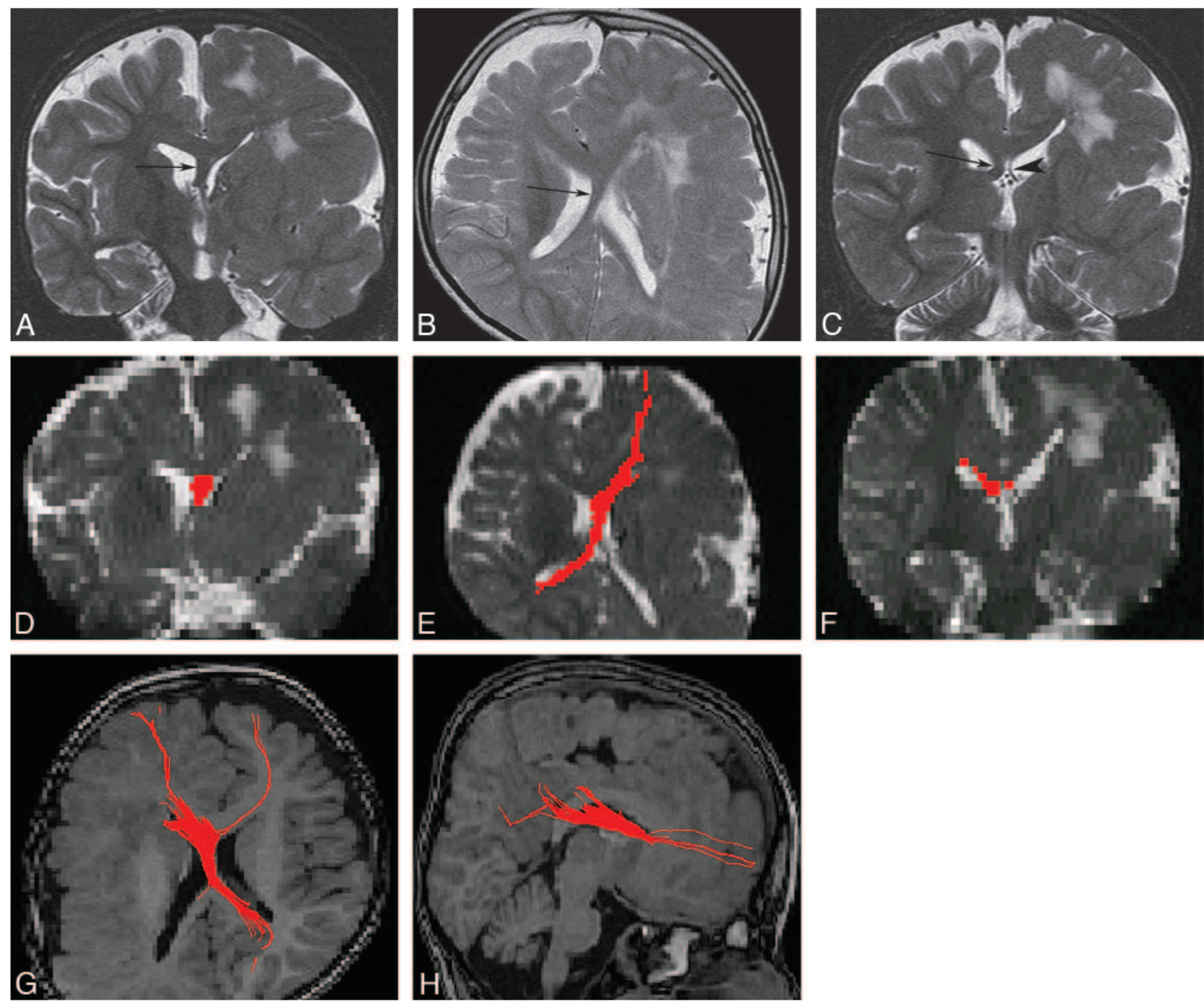

Fig 2. An 8-year-old boy with left hemimegalencephaly (case 1). $A$, Coronal T2-weighted inversion recovery image shows a white matter-intensity structure between the 2 anterior horns (arrow). B, Axial T2-weighted image shows a midsagittal bandlike structure between the bodies of the 2 lateral ventricles (arrow). It appears to cross from anterior-left to posterior-right. $C$, Coronal T2-weighted inversion recovery image. The left fornix (arrowhead) is normal, while the right one (arrow) is thick. $D-F, 2 \mathrm{D}$ coronal $(D), 2 \mathrm{D}$ axial $(E)$, and $2 \mathrm{D}$ coronal $(F)$ views on fiber tract (FT) reconstruction. The images correspond to $A, B$, and $C$, respectively. Aberrant midsagittal fibers penetrate the midsagittal bandlike structures shown in $A-C$. $G$ and $H$, 3D superior $(G)$ and $3 \mathrm{D}$ right lateral $(H)$ views on FT reconstruction. The fibers run between the 2 frontal lobes and the contralateral occipital and parietal lobes. The main fibers pass through the right crus of the fornix.

from 2 to $13 \mathrm{~mm}$ (mean, $5.7 \pm 3.1 \mathrm{~mm}$ ). Asymmetry of the fornix was detected in 7 patients (26.9\%) (Fig 2C), and both fornices were thick in 7 patients (26.9\%) (Fig 3C).

\section{MR Imaging Findings and Diffusion Tensor Tractography in a Prospective Analysis}

In 3 of the 7 patients with hemimegalencephaly, the distance between the 2 anterior horns was not wide, and midsagittal bandlike structures were not observed. The FT reconstruction images for them revealed only portions of the fornices. However, in the remaining 4 patients (cases $1-4$ ), the distance between the 2 anterior horns was wide (from 4 to $22 \mathrm{~mm}$ ) on MR images, and all 4 exhibited aberrant fibers on FT reconstruction images (Figs 2 and 3). In 3 of them, midsagittal bandlike structures beneath the corpus callosum were observed, and their widths were 16,5 , and $3 \mathrm{~mm}$. The asymmetry of the fornix was noted in 2 of them, and both fornices were thickened in 1 patient. For example, in case 1 , on conventional MR imaging, a midsagittal bandlike structure passed between the 2 lateral ventricles and continued to the contralateral asymmetrically thick fornix (Fig $2 A-C$ ). These structures corresponded to aberrant fibers shown on $2 \mathrm{D}$ FT reconstruction images (Fig 2D, $-F$ ). Aberrant fibers ran between the 2 frontal lobes and the contralateral occipital lobe, passing through the contralateral thick crus of the fornix shown on 3D FT reconstruction images (Fig $2 G,-H$ ).

In case 2 , the 2 anterior horns were separated by massive white matter-intensity structures (Fig $3 A$ ), which extended posteriorly and transited into both thick fornices (Fig $3 B,-C$ ). On FT reconstruction images, these structures corresponded to the aberrant fibers shown on $2 \mathrm{D}$ images (Fig $3 D,-F$ ). The 3D FT reconstruction images included all of the aberrant massive fibers, extending between the bilateral frontal lobes and the bilateral occipital and parietal lobes (Fig 3G, -I). In 3D FT reconstruction images of case 3 , aberrant fibers spread to ipsilateral frontal and parietal lobes and the contralateral frontal 

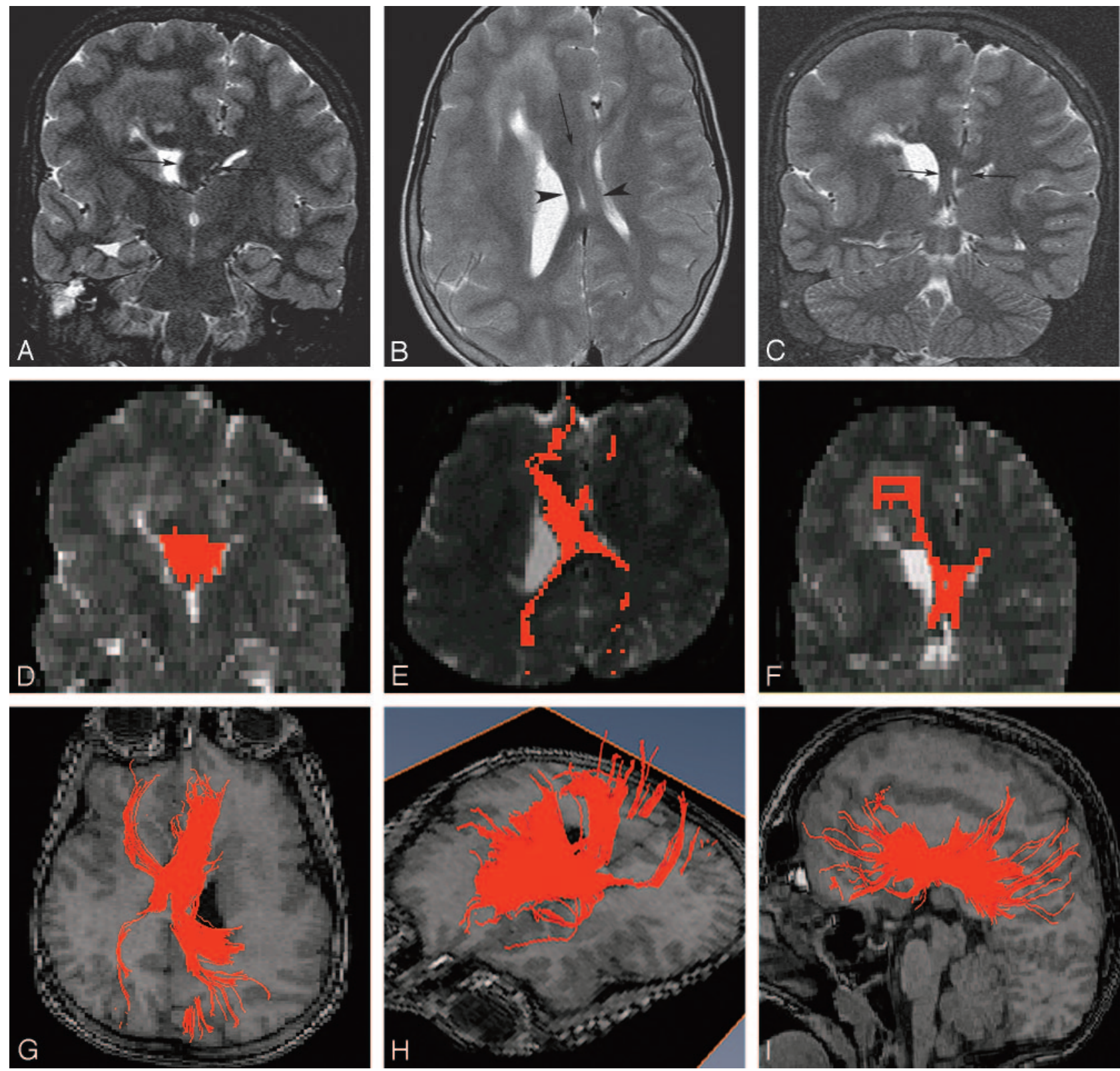

Fig 3. An 11-year-old boy with right hemimegalencephaly (case 2). $A$, Coronal T2-weighted inversion recovery image shows markedly increased width between the 2 anterior horns occupied by a white matter-intensity structure (arrows). B, Axial T2-weighted image demonstrates an abnormal white matter-intensity bandlike structure between the 2 lateral ventricles (arrow), which separates in the posterior portion and runs a course similar to that of the cruces of the fornices (arrowheads). $C$, Coronal T2-weighted inversion recovery image posterior to that in $A$ also detects bilateral marked thickening of the cruces of the fornices (arrows). D-F, 2D coronal $(D), 2 \mathrm{D}$ axial $(E)$, and $2 \mathrm{D}$ coronal $(F)$ views on $\mathrm{FT}$ reconstruction. These images correspond to $A, B$, and $C$, respectively. Aberrant midsagittal fibers penetrate the midsagittal abnormal white matter-intensity structures shown in $A-C$. $G-I, 3 D$ superior $(G), 3 D$ left lateral superior $(H)$, and $3 \mathrm{D}$ left lateral $(\Lambda$ views on FT reconstruction. Aberrant massive fibers connect the bilateral frontal and occipital and parietal lobes passing through abnormal midsagittal structures. They are dominant ipsilaterally.

lobe. In case 4 , aberrant fibers extending to the ipsilateral frontal lobe were crossing to the contralateral crus of the fornix.

\section{Discussion}

Mid-sagittal bandlike structures between the lateral ventricles were observed in more than half of 26 patients with hemimegalencephaly on conventional MR imaging. FT reconstruction images showed them as fibers passing anteroposteriorly through between the 2 anterior horns of the lateral ventricles and connecting intra- or interhemispherically (Figs 2, 3), a finding never observed in healthy subjects. ${ }^{16,17}$

In patients with hemimegalencephaly, only unilateral cere- bral hemisphere is enlarged. Although the etiology of this condition is unknown, it has been postulated to occur due to insults during the second trimester of pregnancy or as early as the third week of gestation, as a genetically programmed developmental disorder related to cellular lineage and establishment of asymmetry. ${ }^{2}$ Hemimegalencephaly may also be a primary disorder of proliferation, in which neurons that are unable to form synaptic connections are not eliminated and thus accumulate. In these processes, normal FTs may not develop or abnormal FTs may develop in abnormal locations.

Aberrant FTs have been reported in other developmental anomalies. ${ }^{18-23}$ The Probst bundle is the most famous of them 
and is observed in patients with agenesis of the corpus callosum. In patients with this condition, during development of axonal fibers (future callosal fibers), the fibers are incapable of crossing the midline due to the absence of the massa commissuralis, and nerve fibers instead follow a caudal course, grouped in a thick longitudinal Probst bundle. Diffusion tensor methods are most suitable for demonstrating it, and many articles on this bundle have been published describing results of diffusion tensor tractography. ${ }^{19-24}$ Vachha et al $^{19}$ described other aberrant fibers in patients with myelomeningocele and Chiari II malformation. They described limbic fiber abnormalities and noted that many patients had defects within the fornices and/or cingulum and that some had aberrant fibers of the cingulum. FT reconstruction images showed fibers of the cingulum crossing obliquely over the corpus callosum and cingulum, which appeared to be composed of multiple parallel aberrant bundles. ${ }^{19}$ In other anomalies, such as holoprosencephaly and lissencephaly, abnormal fibers can also be demonstrated by using diffusion tensor methods. ${ }^{21,23}$ However, there have been no previous reports of diffusion tensor tractography of patients with hemimegalencephaly.

In healthy subjects, no fibers are present at the septum pellucidum, which is located between the 2 anterior horns. ${ }^{16,24}$ However, we found white matter-intensity structures there in $77.4 \%$ of patients with hemimegalencephaly on conventional MR images. In 4 of 7 patients in whom a diffusion tensor study was performed, the distance between the 2 anterior horns was wide $(>4 \mathrm{~mm})$. This region was opacified with white matter intensity, and all 4 had aberrant fibers on FT reconstruction imaging. On the other hand, in 3 of 7 patients, this distance was not wide and no aberrant fibers were detected on FT reconstruction imaging, though portions of the fornices were imaged. The column of the fornix is located in the inferior portion of the septum pellucidum and may be included by placing a region of interest between the anterior horns, or the fornix may be slightly enlarged.

Several articles have described abnormalities of midline structures in patients with hemimegalencephaly detected by imaging. One noted "distortion of the midline,"2 whereas another described "enlarged abnormal gray and white matter structures at the anterior corpus callosum medial to the ventricle that displaced the lateral frontal horns." 25 These findings may correspond to our white matter-intensity structures between the 2 anterior horns. Furthermore, the latter article noted that the incidence of these findings was $75 \%,{ }^{25}$ and almost the same as in our study. However, to our knowledge, no previous study displayed aberrant FTs in patients with hemimegalencephaly.

Our diffusion tensor study included only a limited number of patients, and we have not examined autopsy cases. Further studies with larger groups of patients will yield findings on variation among patients in such aberrant fibers, and autopsy studies would prove the existence of aberrant fibers of patients with hemimegalencephaly.

\section{Conclusions}

The abnormal midsagittal bandlike structures in patients with hemimegalencephaly were revealed as aberrant fibers on FT reconstruction imaging. Such structures were found in more than half of patients with hemimegalencephaly examined. Diffusion tensor imaging is a useful method for demonstrating white matter structures not apparent on routine imaging sequences.

\section{References}

1. Takashima S, Chan F, Becker LE, et al. Aberrant neuronal development in hemimegalencephaly: immunohistochemical and Golgi studies. Pediatr Neurol 1991;7:275-80

2. Flores-Sarnat L. Hemimegalencephaly. Part 1. Genetic clinical and imaging aspects. J Child Neurol 2002;17:373-84

3. Woo CL, Chuang SH, Becker LE, et al. Radiologic-pathologic correlation in focal cortical dysplasia and hemimegalencephaly in 18 children. Pediatr Neurol 2001;25:295-303

4. D’Agostino MD, Bastos A, Piras C, et al. Posterior quadrantic dysplasia or hemi-hemimegalencephaly: a characteristic brain malformation. Neurology 200;62:2214-20

5. Barkovich AJ, Chuang SH. Unilateral megalencephaly: correlation of MR imaging and pathologic characteristics. AJNR Am J Neuroradiol 1990;11:523-31

6. Barkovich AJ, Kuzniecky RI. Neuroimaging of focal malformations of cortical development. J Clin Neurophysiol 1996;13:481-94

7. Kalifa GL, Chiron C, Sellier N, et al. Hemimegalencephaly: MR imaging in five children. Radiology 1987;165:29-33

8. Yagishita A, Arai N, Tamagawa K, et al. Hemimegalencephaly: signal changes suggesting abnormal myelination on MRI. Neuroradiology 1998;40:734-38

9. Sato N, Yagishita A, Oba H, et al. Hemimegalencephaly: a study of abnormalities occurring outside of the involved hemisphere. AJNR Am J Neuroradiol 2007;28:678-82

10. Beaulieu C. The basis of anisotropic water diffusion in the nervous system: a technical review. NMR Biomed 2002;15:435-55

11. Mori S, Crain BJ, Chacko VP, et al. Three-dimensional tracking of axonal projections in the brain by magnetic resonance imaging. Ann Neurol 1999;45:265-69

12. Jiang H, van Zijl PC, Kim J, et al. DtiStudio: resource program for diffusion tensor computation and fiber bundle tracking. Comput Methods Programs Biomed 2006;81:106-16. Epub 2006 Jan 18

13. Pajevic S, Pierpaoli C. Color schemes to represent the orientation of anisotropic tissues from diffusion tensor data: application to white matter fiber tract mapping in the human brain. Magn Reson Med 1999;42:526-40

14. Makris N, Worth AJ, Sorensen AG, et al. Morphometry of in vivo human white matter association pathways with diffusion-weighted magnetic resonance imaging. Ann Neurol 1997;42:951-62

15. Mori S, Kaufman WE, Davatzikos C, et al. Imaging cortical association tracts in the human brain using diffusion-tensor-based axonal tracking. Magn Reson Med 2002; 47:215-23

16. Wakana S, Jiang H, Nagae-Poetscher LM, et al. Fiber tract-based atlas of human white matter anatomy. Radiology 2004;230:77-87

17. Huang $H$, Zhang J, Jiang $H$, et al. DTI tractography based parcellation of white matter: application to the midsagittal morphology of corpus callosum. $\mathrm{Neu}$ roimage 2005;26:195-205

18. Utsunomiya H, Yamashita S, Takano K, et al. Arrangement of fiber tracts forming Probst bundle in complete callosal agenesis: report of two cases with an evaluation by diffusion tensor tractography. Acta Radiol 2006;47:1063-66

19. Vachha B, Adams RC, Rollins NK. Limbic tract anomalies in pediatric myelomeningocele and Chiari II malformation: anatomic correlations with memory and learning — initial investigation. Radiology 2006;240:194-202

20. Lee SK, Mori S, Kim DJ, et al. Diffusion tensor MR imaging visualizes the altered hemispheric fiber connection in callosal dysgenesis. AJNR Am J Neuroradiol 2004;25:25-28

21. Lee SK, Kim DI, Kim J, et al. Diffusion-tensor MR imaging and fiber tractography: a new method of describing aberrant fiber connections in developmental CNS anomalies. Radiographics 2005;25:53-65

22. Hori A, Stan AC. Supracallosal longitudinal fiber bundle: heterotopic cingulum, dorsal fornix or Probst bundle? Neuropathology 2004;24:56-59

23. Rollins N, Reyes T, Chia J. Diffusion tensor imaging in lissencephaly. AJNR Am J Neuroradiol. 2005;26:1583-86

24. Auer RN, Gilbert JJ. Cavum vergae without cavum septi pellucidi. Arch Pathol Lab Med 1982;106:462-63

25. Salamon N, Andres M, Chute DJ, et al. Contralateral hemimicrencephaly and clinical-pathological correlations in children with hemimegalencephaly. Brain 2006;129(Pt 2):352-65. Epub 2005 Nov 16 